carefully examined since if they are normal the diagnosis of coeliac disease must be doubted. Electron microscopy is not necessary for routine diagnosis, while scanning electron microscopy merely provides highly magnified dissecting microscope appearances, though it may prove useful in defining finer degrees of villous abnormality.

In his Oliver-Sharpey lecture, which is concluded at p. 14 of this week's B.M.F., Professor C. C. Booth reviews current knowledge on the pathogenesis of this disease. Gluten is a large molecule and the actual fragment that contains the toxic factor has still to be identified. Toxicity is retained by a mixture of very small peptides, but is lost when they are broken down to their constituent amino-acids. Damage to the intestinal cell results in impairment of its digestive and absorptive functions but the mechanism of the damage is not known. Injured cells are shed prematurely into the intestinal lumen and there is compensatory cell formation in the crypts so that the mucosa is in a hyperregenerative state. Possibly the lysosome is the primary site of intracellular damage and the subsequent release of hydrolytic enzymes wrecks the cell,? but proof that gluten has a predilection for lysosomes is awaited. Gluten may also do more than damage the bowel, since the introduction of a gluten-free diet often results in an immediate improvement in the mental state of the patient, while people with osteomalacia may remain refractory to parenteral vitamin $\mathrm{D}$ therapy until gluten is excluded from the diet.

It has been suggested that coeliac disease results from an inborn error of metabolism in which the intestinal mucosa lacks a peptidase which normally splits and detoxicates gluten. Certainly peptidase activity is reduced in the disease but this appears to be part of a generalized depression of enzyme activity in the damaged mucosa and peptidase levels return to normal after treatment. The alternative theory of aetiology suggests that gluten damages the mucosa by an immune mechanism. Evidence such as the response to steroid therapy, the occurrence of gliadin shock, the delayed response to gluten ingestion, and the presence of lymphocytes and plasma cells in the lamina propria suggests an immunological reaction but is not conclusive. In particular, the presence of antibodies to gluten and other dietary proteins in the serum and jejunal fluid of patients is thought to result from absorption of undigested protein through a damaged mucosa. Nevertheless, evidence of lymphoreticular dysfunction is accumulating; there may be a reduction of lymphoid tissue, impairment of lymphocyte function, changes in the distribution of immunoglobulin-producing cells in the jejunal mucosa, and late development of lymphoma. Changes in the circulating immunoglobulins are harder to assess. Possibly people with an isolated deficiency of IgA (a rare condition) are prone to coeliac disease. ${ }^{8}$ Other workers have reported either an excess of $\operatorname{IgA}^{910}$ or a depression of $\operatorname{IgM}$ in the serum of patients with

\footnotetext{
Rubin, C. E., Eidelman, S., and Weinstein, W. M., Gastroenterology, $1970,58,409$.

2 Mann, J. G., Brown, W. R., and Kern, F., American fournal of Medicine, $1970,48,357$.

${ }^{3}$ Collins, J. R., American Fournal of Digestive Diseases, 1966, 11, 564

3 Collins, J. R., American fournal of Digestive Diseases, 1966, 11, 564. . C., Mortimer, P. E., Stewart, J. S., Nor

s Pollock, D. J., Nagle, R. E., Jeejeebhoy, K. N., and Coghill, N. F., Gut, $1970,11,567$.

6 Pink, I. J., and Creamer, B., Lancet, 1967, 1, 300

7 Riecken, E. O., Stewart, J. S., Booth, C. C., and Pearse, A. G. E., Gut, $1966,7,317$.

\& Crabbé, P. A., and Heremans, J. F., Gut, 1966, 7, 119.

- Eidelman, S., Davis, S. D., Lagunoff, D., and Rubin, C. E., Fournal of Clinical Investigation, 1966, 45, 1003.

10 Asquith, P., Thompson, R. A., and Cooke, W. T., Lancet, 1969, 2, 129.

10 Asquith, P., Thompson, R. A., and Cooke, W. T., Lance,

12 Kenrick, K. G., and Walker-Smith, J. A., Gut, 1970, 11, 635.
}

coeliac disease, ${ }^{11}$ and have noted that the abnormalities disappear on a gluten-free diet. All these findings have recently been confirmed in children and raised IgA levels appear to be common in very young patients with coeliac disease. ${ }^{12}$ These changes in the serum immunoglobulins, except for the IgA deficiency, are secondary to the ingestion of gluten and their role in coeliac disease is still uncertain.

\section{Cholera Again}

The leading article ${ }^{1}$ on "Holiday Cholera" anticipated by less than a week the diagnosis of the disease in a man who returned to Britain from a holiday in Tunisia. Some details of the case are given this week at page 60 . The disease has spread well beyond its usual range, and for the first time this century, and possibly ever, has reached Africa south of the Sahara. Public health authorities in threatened countries are therefore understandably anxious to prevent its entry, and many countries in Europe, Africa, and elsewhere are demanding evidence of vaccination before travellers cross their frontiers. Britain is one of these. ${ }^{2}$ People travelling to this country from any country known to have the disease must produce a valid certificate of vaccination. Those who are going abroad should ascertain whether they will require a certificate to enable them to return to Britain.

The usual vaccination at present is an injection of $0.5 \mathrm{ml}$. of $4,000 \times 10^{6}$ each of killed cholera vibrios of Inaba and Ogawa serotypes per $\mathrm{ml}$. followed 7 to 28 days later by $1 \mathrm{ml}$. of the same. Travellers are allowed to proceed after one injection in most countries. The certificates are valid for only six months. The extent of protection is poorer than with most other immunization procedures and was only $85 \%$ effective three months after the second dose, falling to practically nil by six months. ${ }^{3}$

Cholera is pre-eminently a disease of poor people in poor countries, and it is extremely unlikely to re-establish itself in Britain or the continent of Europe. Africa is clearly at great risk. The infection is generally believed to be waterborne, but this is only partly true. Faecal-oral transmission through the intermediary of food or other means also occurs. It is therefore not sufficient to ensure merely that drinking-water is safe.

Though the disease may infect a considerable proportion of people exposed to it, only about $1 \%$ of those infected have clinical cholera with "rice water stools." 4 A higher and uncertain percentage (possibly up to $10^{\circ}$ ) might have mild diarrhoea or one or more loose motions. The mortality in the severe cases may be up to $70 \%$ or more without treatment.

The cause of death is purely the dehydration and acidosis resulting from the diarrhoea. Patients may lose up to $1,500 \mathrm{ml}$. of fluid per hour, and it is possible for them to die within as little as six to eight hours of the onset of the diarrhoea. In most cases the losses are about 200 to $600 \mathrm{ml}$. per hour, and this rate quickly causes severe dehydration and acidosis. In adults the composition of the stool is a reliable constant, ${ }^{5}$ so that the extent of electrolyte loss from a patient with cholera can be predicted from a knowledge of the stool volume, and determination of the serum and stool electrolytes is unnecessary. In children composition of the stools varies more, but here too it is possible to predict losses of electrolyte sufficiently accurately to determine treatment. ${ }^{\text {' }}$

The disease is self-limiting. The diarrhoea usually lasts 
four to five days in the untreated case and about a day less in those treated. All patients alive on arrival at a treatment centre should recover if given appropriate fluids sufficiently quickly and in sufficient quantity.

There are two phases of treatment-the correction of dehydration and electrolyte imbalance, and the maintenance of this correction. Intravenous fluid (the 5/4/1 Dacca solution) of the following composition serves for both: $\mathrm{NaCl}_{5} \mathrm{~g}$., $\mathrm{NaHCO}_{3} 4 \mathrm{~g}$., $\mathrm{KCl} 1 \mathrm{~g}$., distilled water to 1 litre. ${ }^{7}$

In the correction phase the patients are severely dehydrated and acidotic, but it is sufficient to determine the degree of dehydration alone by a simple procedure such as the measurement of plasma specific gravity (P.S.G.) by the copper sulphate drop method of Phillips and colleagues. ${ }^{8}$ The amount of intravenous fluid required can then be calculated according to the formula (Estimated P.S.G. minus 1.025 ) $\times 200,000=\mathrm{ml}$. fluid required. For example, the estimated P.S.G. is 1.037 . Subtraction of 1.025 leaves 0.012 . Multiplication of this by 200,000 gives 2,400 , and this is the required amount of fluid in ml. The fluid must be given intravenously as fast as is possible, usually within half an hour or less.

When polyethylene catheters are available for intravenous use, it is convenient to insert one for infusion, but the administration of fluid must not be delayed through waiting for the catheter: infusion should be started through a needle and a change made later if necessary. A particular advantage of the polyethylene catheter is that it may be used for measuring the central venous pressure (C.V.P.), and this in turn may be used to assess the adequacy of fluid replacement instead of the plasma specific gravity. After approximately 1 litre of the infusion fluid has been given as rapidly as possible the patient is propped up in bed with the upper half of the body at $45^{\circ}$ from the horizontal. The drip is then stopped and disconnected from the infusion set, and the polyethylene tube is held vertically alongside the thorax. The fluid in it will level off at the central venous pressure. If the pressure is more than $5 \mathrm{~cm}$. below the sternal angle, further fluid should be infused until the pressure is $1-2 \mathrm{~cm}$. below the sternal angle. At this point the hydration can be assumed to be adequate, and further infusion of fluid is regulated to balance losses as indicated below. Fast infusion of fluid is safe so long as the central venous pressure does not rise above the sternal angle. Above this level there is a danger of inducing pulmonary oedema.

Maintenance therapy is simple. The stool volume should be measured hourly and the rate of infusion of the same mixture regulated to equal it. Vomiting almost invariably ceases with the passage of the first few watery stools, and the patient should be given water and glucose-containing drinks ad libitum plus $1-2 \mathrm{~g}$. tetracycline per day. ${ }^{9}$

In countries where the disease may occur in epidemic proportions the very large amounts of intravenous fluids required ( 20 or more litres per patient) may put a strain on supplies. It is therefore important to know that recently a regimen of treatment by mouth has been developed at the

British Medical fournal, 1970, 3, 601

2 C. M. O. Circular 13/70.

Mosley, W. H., et. al., Bulletin of the World Health Organization, 1969, 40, 187 - Mosley, W. H., et al., Bulletin of the World Health Organization, 1969,
40,187.

- Phillips, R. A., Federation Procecdings, 1964, 23, 705.

- Rahaman, M. M., Rahman, W., Hare, W. K., Hare, R. D., and Phillips, R. A. Eighth International Congress of Tropical Medicine, Abstracts and Reviews, 1968, p. 490.

Nalin, R., et al., Lancet, 1968, 2, 370.

* Phillips, R. A., et al., Fournal of Biological Chemistry, 1950, 183, 305

- Pierce, N. F., et al., British Medical fournal, 1968, 3, 277.

- Cash, R. A., Nalin, D. R., Forrest, J. N., and Abrutyn, E., Lancet, 1970, 2, 549

$"$ P.S.C.R.L. Technical Committee Report, 1969.
Cholera Research Laboratories established under the joint auspices of the South-east Asia Treaty Organization and Pakistan. It may be used in the maintenance phase of treatment with substantial saving of intravenous fluid and may even be used alone in mild cases. ${ }^{10}$ The success of this regimen depends on the fact that glucose given by mouth enhances absorption of sodium. Glycine even further enhances absorption of sodium, and treatment incorporating this as well as glucose and electrolytes in the fluid has been under trial in the same laboratories, with encouraging results. ${ }^{11}$ It is here fitting to pay a tribute to Dr. R. A. Phillips and his team at those laboratories, for they have made substantial contributions to the understanding of this disease and its management.

\section{Renal Glomerular Disease}

Similar symptoms can arise from a variety of pathological changes in the renal glomeruli. It is not surprising, therefore, that earlier classifications of glomerulonephritis-such as that of A. Ellis ${ }^{1}$ - which were based on clinical and necropsy studies, lost their relevance when the use of renal biopsy enabled the structure of the kidneys to be examined during life. A complete diagnosis in kidney disease requires a description of the clinical syndrome, of the renal lesions, and, when known, of the cause of the condition. But correlations between clinical conditions and pathological lesions are now well enough known to allow us to divide primary glomerular disease on a pathological basis into several categories. These are not necessarily discrete entities, but each is sufficiently homogeneous to be useful in determining the prognosis for individual patients and in providing a guide to treatment.

Such a classification has been summarized recently by J. Churg, R. Habib, and R. H. R. White. ${ }^{2}$ They described five major groups-minimal change, focal glomerulosclerosis, membranous (epimembranous) nephropathy, proliferative glomerulonephritis, and chronic glomerulonephritis. The last group represents the end stage of many conditions, and glomerular destruction is too advanced for the recognition of specific features.

Proliferative glomerulonephritis comprises an assortment of pathological conditions and has five subdivisions. These are: the diffuse exudative lesion, characteristic of the acute phase of post-streptococcal glomerulonephritis; mesangial proliferation, which is seen in subsiding post-streptococcal glomerulonephritis but which also occurs without evidence of previous streptococcal infection; focal proliferation, often associated with the Henoch-Schonlein syndrome or other multi-system diseases; epithelial crescents, which, when large, cause obliteration of glomeruli, leading to rapidly progressive renal failure; and membranoproliferative glomerulonephritis. The renal lesions of multi-system diseases, such as systemic lupus erythematosus, polyarteritis nodosa, subacute bacterial endocarditis, and Goodpasture's syndrome, may be indistinguishable from those of primary proliferative glomerulonephritis. Most of the categories are familiar, but in two cases-focal glomerulosclerosis and membranoproliferative glomerulonephritis-the associations between clinical and pathological features have only recently been clarified.

In focal glomerulosclerosis (found in about $9^{\circ} \prime$, of children with the nephrotic syndrome ${ }^{2} 3$ ) totally or segmentally sclerosed glomeruli are interspersed with normal glomeruli, the juxtamedullary glomeruli being involved earliest. ${ }^{4}$ The condition may be difficult to distinguish on renal biopsy from 